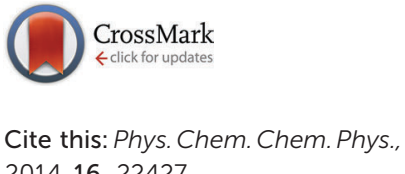

$2014,16,22427$

DOI: $10.1039 / c 4 c p 90130 d$

\title{
Correction: Trajectory analysis of single molecules exhibiting non-Brownian motion
}

\author{
Lindsay C. C. Elliott, ${ }^{a}$ Moussa Barhoum, ${ }^{b}$ Joel M. Harris*b and Paul W. Bohn*c \\ Correction for 'Trajectory analysis of single molecules exhibiting non-Brownian motion' by Lindsay \\ C. C. Elliott et al., Phys. Chem. Chem. Phys., 2011, 13, 4326-4334.
}

www.rsc.org/pccp

It has been brought to our attention that an error exists in our 2011 paper, 'Trajectory analysis of single molecules exhibiting non-Brownian motion'.

The problem is in the definition of the eigenvalues, and just after eqn (2) on p. 4328 (3rd page of the article) should read: “... where $R_{1}^{2}$ and $R_{2}^{2}$ are the major and minor eigenvalues, respectively, of the radius of gyration tensor, $\boldsymbol{T}$." This definition permits correct calculation of the radius of gyration, $R_{\mathrm{g}}$, and allows the units to work out as you would expect, with $R_{\mathrm{g}}$ in meters.

The Royal Society of Chemistry apologises for these errors and any consequent inconvenience to authors and readers.

\footnotetext{
${ }^{a}$ Department of Chemistry, University of Illinois at Urbana-Champaign, 600 S. Mathews Ave., Urbana, IL 61801, USA. E-mail: pbohn@nd.edu, harrisj@chem.utah.edu

${ }^{b}$ Department of Chemistry, University of Utah, 315 South 1400 East, Salt Lake City, UT 84112, USA

${ }^{c}$ Department of Chemical and Biomolecular Engineering and Department of Chemistry and Biochemistry, University of Notre Dame, Notre Dame, IN 46556, USA
} 